\title{
Integration of small RNAs, degradome, and transcriptome sequencing provides insights into the differences between Shizhu ginseng and Yuan ginseng
}

\author{
Mengyuan Peng ${ }^{1} \cdot$ Yanlin Chen ${ }^{1} \cdot$ Wenjuan Wang ${ }^{1} \cdot$ Zhihua He $^{1} \cdot$ Wangqiang Dai $^{1} \cdot$ Zhiyun Lin $^{1} \cdot$ \\ Zemin Yang ${ }^{3} \cdot$ Mengjuan Gong ${ }^{1} \cdot$ Yongqin Yin $^{1} \cdot \operatorname{Bin} \mathrm{Han}^{1} \cdot$ Yu Zeng ${ }^{1} \cdot$ Yingfang Wang $^{1,2}$
}

Received: 10 September 2019/Accepted: 21 September 2020/Published online: 30 October 2020

(c) The Author(s) 2020

\begin{abstract}
Panax ginseng is one of the most popular herbs which have been used as an important traditional Chinese medicine since ancient times. Yuan ginseng and Shizhu ginseng,which belong to $P$. ginseng, are widely used as substitutes for wild ginseng in clinical practice. Clinical practice has proved that the clinical efficacy of Shizhu ginseng is better than Yuan ginseng. However, current research cannot completely explain this phenomenon. Considering that small RNA may be one of the pharmacodynamic substances of $P$. ginseng, it is challenging to investigate differential miRNAs between Shizhu ginseng and Yuan ginseng. In this study, the transcriptome, small RNAome and degradome of $P$. ginseng were studied by high-throughput sequencing. A total of 63,875 unigenes and 43,950,137 small RNA clean reads were obtained from the roots of $P$. ginseng. Among 3206 differentially expressed genes, 1190 genes were up-regulated in Yuan ginseng when compared with Shizhu ginseng. 24 known differential miRNAs and 7 novel differential miRNAs were obtained. The 304 targets of 24 differentially expressed miRNA (17 known and 7 novel) families are mainly related to energy metabolism, biotic stress and disease immunity in ginseng itself. Through the association analysis of mRNA and miRNA, our work gives a better understanding of the difference between Yuan ginseng and Shizhu ginseng. Considering the cross-kingdom regulation of plant miRNAs, our results may provide a foundation for understanding the miRNA-dependent clinical efficacy in $P$. ginseng.
\end{abstract}

Keywords Yuan ginseng $\cdot$ Shizhu ginseng $\cdot$ miRNA $\cdot$ Target gene $\cdot$ High-throughput sequencing

\begin{tabular}{|c|c|c|c|}
\hline \multicolumn{2}{|c|}{ Abbreviations } & NCBI & National Center for Biotechnology \\
\hline \multicolumn{2}{|c|}{ miRNA microRNA } & & Information \\
\hline \multicolumn{2}{|r|}{ Untranslated region } & NR & NCBI non-redundant \\
\hline \multicolumn{2}{|c|}{ SRA } & KOG & EuKaryotic Orthologous Groups \\
\hline & & KEGG & Kyoto Encyclopedia of Genes and Genomes \\
\hline & & $\mathrm{GO}$ & Gene ontology \\
\hline \multirow{4}{*}{\multicolumn{2}{|c|}{$\begin{array}{l}\text { Electronic supplementary material The online version of this } \\
\text { article (https://doi.org/10.1007/s13562-020-00613-5) con- } \\
\text { tains supplementary material, which is available to autho- } \\
\text { rized users. }\end{array}$}} & FPKM & Reads per kb per million reads \\
\hline & & DEGs & Differentially expressed genes \\
\hline & & $\mathrm{FC}$ & Fold change \\
\hline & & TPM & Transcript per million \\
\hline \multirow[t]{2}{*}{ 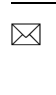 } & Yingfang Wang & DEMs & Differentially expressed miRNAs \\
\hline & 150306757@qq.com & qRT-PCR & Quantitative real-time PCR \\
\hline \multirow[t]{2}{*}{1} & & RIN & RNA integrity number \\
\hline & $\begin{array}{l}\text { School of I raditional Chinese Mledicine, Guangdong } \\
\text { Pharmaceutical University, Guangzhou 510006, China }\end{array}$ & TIR1 & Transport inhibitor response 1 \\
\hline \multirow{3}{*}{2} & & GRF & Growth-regulating factor \\
\hline & $\begin{array}{l}\text { Guangdong Engineering and Technology Research Center of } \\
\text { Topical Precise Drug Delivery System, Guangzhou 510006, }\end{array}$ & GATLA & Glycosyltransferase \\
\hline & China & LDLRAP1 & Low density lipoprotein receptor adapter \\
\hline 3 & $\begin{array}{l}\text { School of Basic Courses, Guangdong Pharmaceutical } \\
\text { University, Guangzhou 510006, China }\end{array}$ & EVs & $\begin{array}{l}\text { protein } 1 \\
\text { Extracellular vesicles }\end{array}$ \\
\hline
\end{tabular}




\begin{tabular}{|c|c|}
\hline LU7L3 & luc7-like protein 3 isoform $\mathrm{X} 2$ \\
\hline TBCD & Tubulin-folding cofactor D \\
\hline Dscam & Down's syndrome cell adhesion molecule \\
\hline B561A & $\begin{array}{l}\text { Cytochrome b561, DM13 and DOMON } \\
\text { domain-containing protein }\end{array}$ \\
\hline TIR & Toll-interleukin-like receptor, nucleotide- \\
\hline NBS-LRR & $\begin{array}{l}\text { binding site and C-terminal leucine-rich } \\
\text { repeat }\end{array}$ \\
\hline ICE1 & $\begin{array}{l}\text { Basic helix-loop-helix (bHLH) DNA- } \\
\text { binding superfamily protein }\end{array}$ \\
\hline
\end{tabular}

\section{Introduction}

Ginseng is the dry root and rhizome of Panax ginseng C.A. Mey, which belongs to the Panax genus in Araliaceae family. It has widely been used as an important traditional medicine for thousands of years in Eastern Asia, especially China. Evidence from clinical trials demonstrated that $P$. ginseng could play significant roles in many human diseases such as cancer, neurodegenerative diseases (Rajabian et al. 2019) and so on. Owing to its important medicinal properties, $P$. ginseng has been highly valued and has been called "the king of herbs". Because $P$. ginseng is consumed in large quantities and grows very slowly in natural conditions, wild-grown ginseng cannot meet the growing demand from the medical market. Consequently, $P$. ginseng was artificially cultivated to meet demand for this precious herb and cultivation of this herb dates back at least as far as the Ming dynasty. According to different growth years, cultivated ginseng can be divided into Shizhu ginseng and Yuan ginseng. Yuan ginseng is a ginseng artificially cultivated in gardens or fields for $6-8$ years. Shizhu ginseng is a ginseng cultivated in a special method for 10-20 years, and its medicinal value is comparable to that of wild ginseng.

Although Shizhu ginseng and Yuan ginseng belong to cultivated ginseng, years of medical practice have shown that the clinical efficacy of Shizhu ginseng is significantly better than that of Yuan ginseng. To date, modern pharmacological research has focused on the ginsenosides and polysaccharides, the major bioactive compound of $P$. ginseng. However, it cannot completely explain the reasons for the huge difference in clinical efficacy between Shizhu ginseng and Yuan ginseng (Chunlu et al. 2016; Keqiang et al. 2013).

MicroRNAs (miRNAs) are a class of endogenous single-stranded RNAs of about $20 \mathrm{nt}$ to $24 \mathrm{nt}$ that can be paired with the $3^{\prime}$-untranslated region (UTR) of a target gene in a complete or incomplete manner. It can regulate gene expression at post-transcriptional levels (Dong et al. 2013), which plays a major role in lots of aspects (Yingfang et al. 2019). MiRNA research technology such as small RNA sequencing has been widely used in clinical and basic research which has become an important means for scientists to study the function of miRNAs. The transcriptome reflects the expression of specific cell or tissue genes at a specific development or physiological stage. Usually, differentially expressed genes (DEGs) were combined with differentially expressed miRNAs (DEMs) to analyze the regulatory mechanisms in which miRNAs might be involved. Degradome sequencing, as a highthroughput experimental method for identifying the target transcripts of plant miRNAs (Wang et al. 2015), can be employed to verify the miRNA target genes in plants accurately on a large scale (Mathiyalagan et al. 2013).

In this study, high-throughput sequencing technology was used to compare the differences of miRNAs contained in Shizhu ginseng and Yuan ginseng. In order to explore the mechanism of the difference in clinical efficacy of ginseng with different growth years, we began with the biological functions of non-coding miRNAs contained in Shizhu ginseng and Yuan ginseng.

\section{Method}

\section{Plant material}

The 15-year-old Shizhu ginseng and the 6-year-old Yuan ginseng were all collected from the ginseng market of Changbai Mountain in Wanliang Town, Fusong County, Jilin Province, China. They were identified as Panax ginseng C.A. Mey by the Associate Professor Hongyan Ma from the School of Traditional Chinese Medicine of Guangdong Pharmaceutical University. The main roots of ginseng were cleaned with distilled water, cut into small pieces, and then immediately frozen in liquid nitrogen until further processing.

\section{RNA extraction}

The total RNA was extracted by using Trizol reagent (Invitrogen) according to the manufacturer's protocols, and $100 \mathrm{mg}$ of snap-frozen Shizhu ginseng and Yuan ginseng root tissue was used, respectively. The quantity and purity of the total RNA were measured by $1 \%$ agarose gel electrophoresis, NanoDrop 2000 spectrophotometer and Agilent 2100 Bioanalyzer (RNA integrity number (RIN) $>7.0,28 \mathrm{~S} / 18 \mathrm{~S} \geq 0.7)$. For each of two samples, at least three individual ginseng roots were collected for RNA 
isolation. The mixed total RNA from Yuan ginseng or Shizhu ginseng was used for library construction for transcriptome, small RNA, and degradome sequencing. The library construction and sequencing were conducted by $\mathrm{OE}$ Biotech (Shanghai, China).

\section{Library construction and high-throughput sequencing for transcriptome, small RNA and degradome}

The high-quality total RNA was used for transcriptome library construction using Illumina's mRNA-Seq sample preparation kit. Briefly, mRNA was isolated from total RNA using oligo-dT magnetic beads and fragmented, followed by cDNA synthesis. The cDNA fragments were amplified to generate transcriptome libraries which were sequenced on Illumina HiSeq 2500 platform.

Two libraries were constructed using the extracted RNA according to standard procedures for small RNA library construction and sequencing. In brief, high-quality RNA from each sample was separated by polyacrylamide gel electrophoresis. RNA fragments of length 18-30 nt were enriched and were ligated to $3^{\prime}$ and $5^{\prime}$ adapter. Then the fragments were $t$ subjected to cDNA synthesis, followed by PCR amplification and sequencing on Illumina HiSeq 2500.

The degradome library construction was performed as previously described (Garg et al. 2019) followed by sequencing.

\section{Analysis of transcriptome sequencing data}

The raw data obtained from sequencing all these samples were processed to remove adaptor contamination and lowquality reads using FASTQC (Cock et al. 2010). The Trinity software was used to assemble the unigene sets from the clean reads of Yuan ginseng and Shizhu ginseng, using the default parameters. After short-sequence assembly and splicing, non-all-unigene was obtained. Then it was compared with NCBI non-redundant (NR), EuKaryotic Orthologous Groups (KOG), Kyoto Encyclopedia of Genes and Genomes (KEGG), Gene Ontology (GO) and other databases to obtain the protein functional annotation and metabolic pathway information of unigene. The gene expression level for each transcript was calculated by FPKM (reads per kb per million reads). Differentially expressed genes (DEGs) were identified between different samples by using DESeq software (Kvam et al. 2012). A gene was considered to be differentially expressed between two samples if it exhibited fold change (FC) value of $\geq 2$ (up-regulation) or $\leq 0.5$ (down-regulation) with the $p$ value $\leq 0.05$. GO functional enrichment and KEGG pathway analyses were performed, respectively, to understand the function of DEGs.

\section{Analysis of small RNA sequencing data}

Adapters were removed and low-quantity sequences were filtered from raw data to obtain clean reads. The clean reads were aligned with the Rfam, Gene, Repbase to exclude rRNA, tRNA, snRNA, snoRNA, and other ncRNA, and repeats. The remaining reads were aligned with miRBase 21.0 databases to map known miRNAs, and the unpaired sequences were used for the prediction of novel miRNAs using miRDeep2 software (Mackowiak 2011). The expression levels of known miRNAs and newly predicted miRNAs were calculated by TPM (transcript per million). Differentially expressed miRNAs (DEMs) were identified with FC $>2$ (up-regulation) or $\mathrm{FC}<0.5$ (downregulation) and the threshold of $p$ value $<0.05$. The $p$ value was calculated with Audic Claverie statistic. miRNA targets were predicted from the assembled $P$. ginseng unigene set by the psRNATarget server using default parameters. GO annotation and KEGG pathway analysis were performed on the target genes of DEMs.

\section{Analysis of degradome sequencing data}

Clean tags were obtained by data filtering. After annotation of Rfam and Genbank database and identification of Poly $\mathrm{N}$,functional annotations for clean tags were obtained. The remaining tags were compared with reference genome to obtain cDNA-sense. The tags (cDNA-sense) which positively aligned to the reference genome (unigenes) in the clean tags were selected to predict and analyze the miRNA degradation sites. Cleaveland 3 software was used for identification and annotation of miRNA cleavage sites. The reference gene sequence in which the predicted degradation site is located is aligned with the Nr, KEGG, and GO databases to obtain functional annotation information of the degraded gene.

\section{Validation of DEMs and DEGs expression by qRT- PCR}

17 DEGs and 9 DEMs of interest were randomly selected for quantitative real-time PCR (qRT-PCR) analysis. The total RNA was extracted by using the Trizol reagent, then it was determined using a NanoDrop 2000 spectrophotometer and agarose gel electrophoresis stained with ethidium bromide. Quantification was performed with a two-step reaction process: reverse transcription (RT) and PCR. qRTPCR analysis was carried out on LightCycler ${ }^{\circledR} 480$ II Realtime PCR Instrument (Roche, Swiss) using QuantiFast ${ }^{\circledR}$ SYBR $^{\circledR}$ Green PCR Master Mix (Qiagen, Germany). Each 
sample was run in triplicate for analysis. The expression levels of mRNAs and miRNAs were normalized to $18 \mathrm{~S}$ rRNA and U6 respectively, and were calculated using the $2^{-\Delta \Delta \mathrm{Ct}}$ method.

\section{Results}

\section{Total RNA quality, concentration and integrity testing}

Agarose gel electrophoresis showed that the $28 \mathrm{~S}$ and $18 \mathrm{~S}$ bands of the two samples were clear and complete (Fig. 1), indicating that the extracted RNA was in good integrity. And the total RNA band brightness of the Yuan ginseng was twice as bright as that of the total RNA band of the Shizhu ginseng. This may be due to the long growth period of the Shizhu ginseng that enriched secondary metabolites such as polysaccharide and polyphenols.

The Agilent 2100 Bioanalyzer was used to perform a quality check on the RNA indicators (Table S1). The concentrations of Shizhu ginseng and Yuan ginseng were $909.24 \mathrm{ng} \mu \mathrm{L}^{-1}$ and $1008.79 \mathrm{ng} \mu \mathrm{L}^{-1}$, respectively. RNA integrity number (RIN) of Shizhu ginseng and Yuan ginseng was 9.7 and 8.8 , respectively. $28 \mathrm{~S} / 18 \mathrm{~S}$ of Shizhu ginseng and Yuan ginseng was 1.9 and 1.8, respectively. The results showed that the total RNA quality met the criteria for high-throughput database construction and the Illumina HiSeq ${ }^{\mathrm{TM}} 2500$ sequencing.

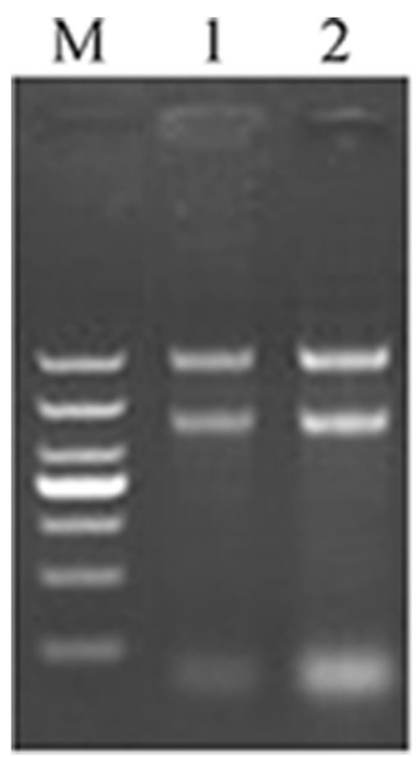

\section{$28 \mathrm{~S}$}

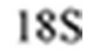

$5 \mathrm{~S}$

Fig. 1 RNA electrophoresis. M: 1000 marker; 1: total RNA of Shizhu ginseng; 2: total RNA of Yuan ginseng

\section{DEGs between Shizhu ginseng and Yuan ginseng}

To identify transcripts that are involved in different growth year of $P$. ginseng, RNA-seq libraries from Shizhu ginseng and Yuan ginseng were constructed. Approximately 52.5 million raw reads were obtained from each RNA-seq library (Table S2). A total of 104.97 million reads were generated from the paired-end sequencing of these 2 samples. After the stringent quality filters, $94.8 \%$ (99.51 million) of the reads representing high-quality reads $(\mathrm{Q} 30)$ were processed for further analysis. Then the high-quality reads were used for de novo assemble and 63,875 unigenes were obtained.

According to the differential gene screening method in Method, 3216 differentially expressed genes (DEGs) were obtained. 1190 genes were up-regulated in the Yuan ginseng compared to those of Shizhu ginseng, while 2026 genes were down-regulated (Fig. 2, Table S3). To verify DEGs identified by RNA-Seq,17 DEGs were randomly selected for qRT-PCR verification. The results showed the same expression patterns with RNA-Seq data (Fig. S1).

\section{Functional analysis of DEGs}

A gene entry satisfying the condition of $p$ value $\leq 0.05$ and $\mathrm{FC}>2$ or $\mathrm{FC}<0.5$ is defined as GO Term. 1190 upregulated DEGs were enriched into 678 GO Terms, and 2016 down-regulated DEGs were enriched into 1153 GO Terms. Within GO Term analysis, DEGs which were up regulated in the Yuan ginseng mainly related to

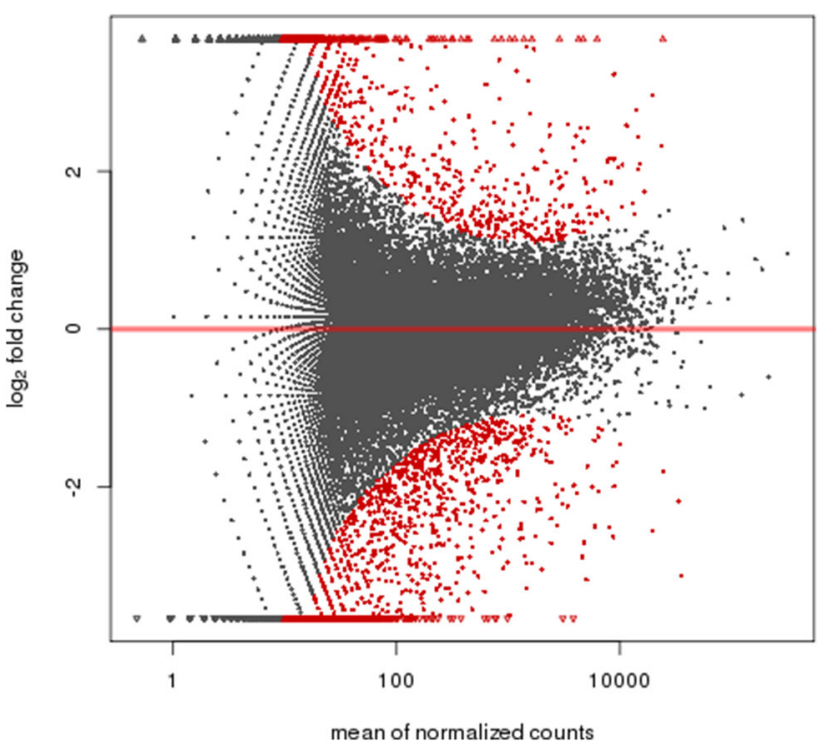

Fig. 2 Volcano map of DEGs. The X-axis is the average of all sample expressions used for comparison after normalization, and the $\mathrm{Y}$-axis is $\log 2$ fold change. Red is marked with a difference of significant $p$ value $<0.05$ unigene 
transcription DNA-templated, monolayer-surrounded lipid storage body, transcription factor activity, etc. The DEGs which down-regulated in the Yuan ginseng were enriched in the unsaturated fatty acid biosynthetic process, apoplast, heme binding, and so on (Fig. 3).

To further understand the function of DEGs between Shizhu ginseng and Yuan ginseng, we performed KEGG analysis. KEGG pathway enrichment analysis of DEGs was done based on hypergeometric model with a significance threshold of $p$ value 0.05 . Most of the significantly enriched up-regulated DEGs belong to the MAPK signaling pathway, photosynthesis-antenna proteins, spliceosome and so on. Down-regulation of DEGs was significantly enriched in pathways such as phenylpropanoid biosynthesis, biosynthesis of unsaturated fatty acids and fatty acid metabolism, etc. (Figure 4).

\section{Identification of differentially expressed miRNAs (DEMs) among Shizhu ginseng and Yuan ginseng}

The raw data obtained by high-throughput small RNA sequencing was purified to obtain clean reads, and the length distribution of small RNAs with a length of 18-41 nt was statistically analyzed. The small RNA sequences of Shizhu ginseng and Yuan ginseng were enriched at the length of $24-21 \mathrm{nt}$ (51.64\% and $66.03 \%$, respectively), indicating that miRNA is the main fragment in the deep sequencing results, which provides a powerful basis for the analysis of miRNA.

Differential expression analysis between Shizhu ginseng and Yuan ginseng was performed. DEMs were selected with FC $>2$ or $<0.5$ and $p$ value $\leq 0.05$ by TPM statistic.
For known miRNAs, 24 DEMs were obtained, 13 of which were up-regulated and 11 were down-regulated (Fig. 5, Table S4). A total of 39 novel miRNAs (21 new miRNA hairpin precursors and 18 miRNA* $^{*}$ sequences) were obtained through prediction (Table S5). For novel miRNAs, a total of 7 differential miRNAs were obtained, of which 5 novel miRNAs were up-regulated in the ginseng and 2 were down-regulated (Fig. 6). 7 DEMs were randomly selected for qRT-PCR verification and the results showed consistence with small RNA sequencing data (Fig. S2).

\section{Target gene prediction and analysis of DEMs}

Plant miRNAs show perfect or near-perfect complementarities to their targets. It allows an effective prediction of miRNA targets through computation. To predict the targets of DEMs in $P$. ginseng, we used psRNATarget (Dai et al. 2018), PMRD (Zhang et al. 2010) and TAPIR (Bonnet et al. 2010) and other biological software. We identified a total of 304 unigenes from 63,875 assembled $P$. ginseng unigenes to be targets of 17 known miRNA families and 7 novel miRNAs (Table 1).

GO and KEGG functional enrichment analysis was performed on 197 target genes of 17 known miRNA families, and a total of $260 \mathrm{GO}$ functions and 36 pathway pathways were annotated. The GO analysis results contain three parts: biological process, cellular component, and molecular function, and all the most strongly enriched elements are shown in Fig. 7a. Within biological process, regulation of transcription, cell differentiation, leaf development and auxin-activated signaling pathway were the

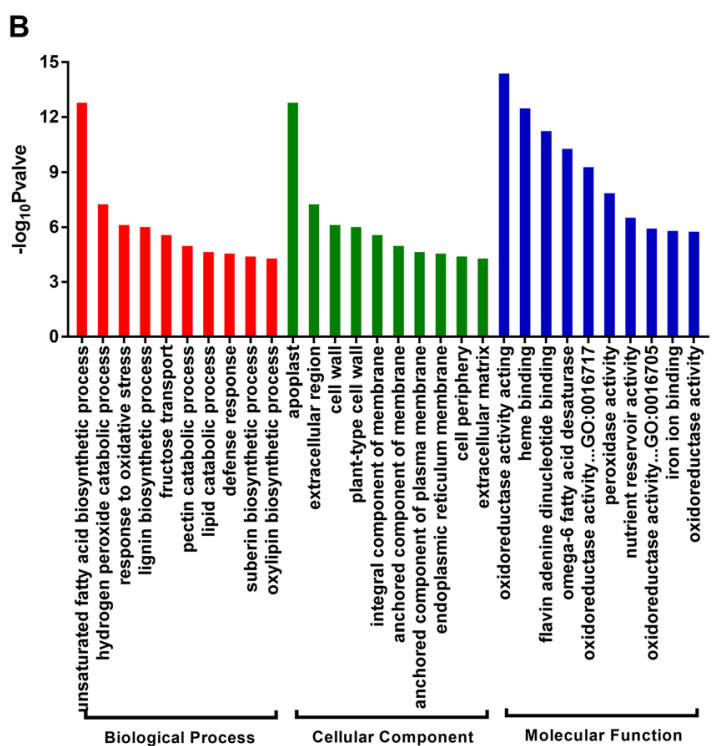

Fig. 3 The GO functional analysis of DEGs. a The GO functional analysis of up-regulated DEGs. b The GO functional analysis of downregulated DEGs 
A

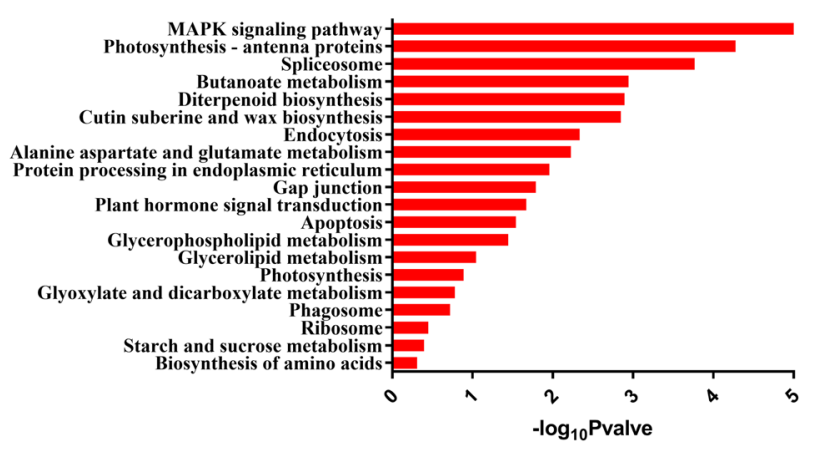

B

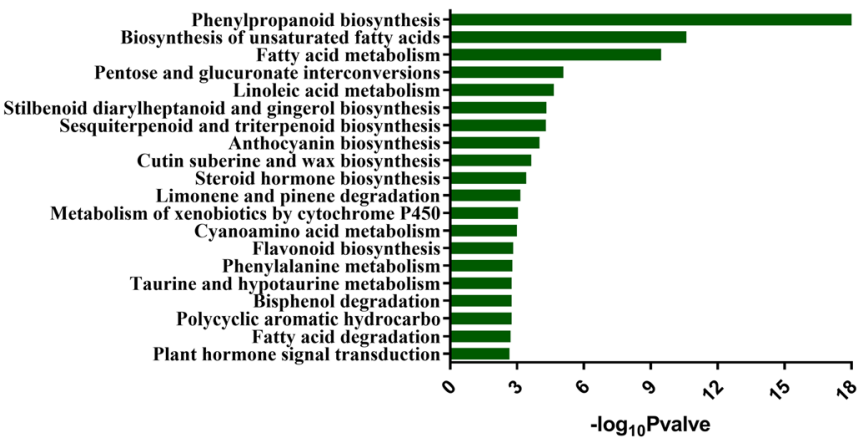

Fig. 4 The KEGG analysis of differential expressed genes. a The KEGG analysis of up-regulated genes. b The KEGG analysis of downregulated genes

A

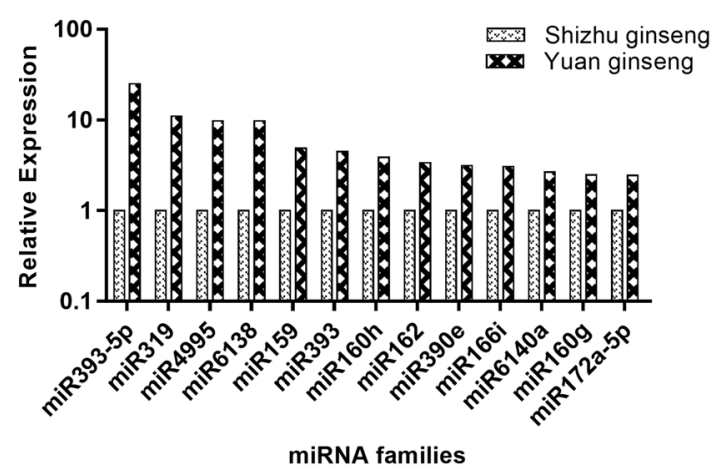

B

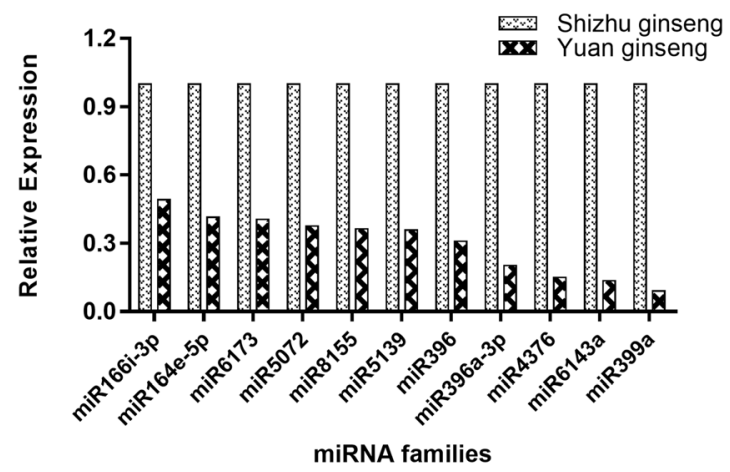

Fig. 5 The relative expression level of known differential miRNAs. a The relative expression level of up-regulated miRNAs in Yuan ginseng. b The relative expression level of down-regulated miRNAs

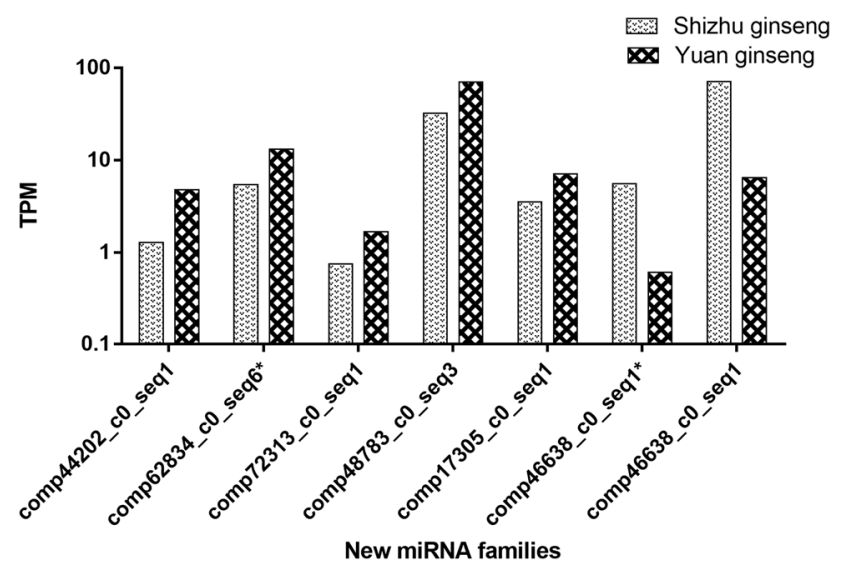

Fig. 6 Differential expression of novel miRNAs

most significantly enriched elements; within cellular component, the most significantly enriched element were nucleus, integral component of membrane, and cytoplasm; and within molecular function, binding, catalytic activity, and transcription factor activity groups were the most significantly enriched elements.
Through KEGG Pathway functional enrichment analysis, it is known that differential miRNA target genes are enriched in plant hormone signal transduction, Wnt signaling pathway, ubiquitin mediated proteolysis and so on (Fig. 7c).

For target genes of 7 novel miRNAs, we performed KEGG and GO enrichment analysis. A total of 167 GO functional categories and 37 KEGG pathways were annotated. The results of GO analysis have been showed in Fig. 7b Within biological process, regulation of transcription, transcription (DNA-templated) and root development were the most significantly enriched elements; within cellular component, the most significantly enriched element were nucleus, integral component of membrane and plasmodesma; and within molecular function, binding, catalytic activity and transcription factor activity groups were the most significantly enriched elements. These GO functional categories of novel miRNA target gene were very similar to those predicted by known differential miRNAs.

KEGG Pathway functional enrichment showed that the target genes of novel miRNAs were enriched in plant hormone signal transduction, p53 signaling pathway, metabolism, ubiquitin mediated proteolysis (Fig. 7d). This 
Table 1 Predicted targets of Panax ginseng miRNAs

\begin{tabular}{|c|c|}
\hline miRNA & Target gene \\
\hline \multicolumn{2}{|l|}{ Known-miRNAs } \\
\hline $\operatorname{miR} 159$ & MYB, ACBP5 \\
\hline $\operatorname{miR} 160$ & ARF \\
\hline $\operatorname{miR} 162$ & DCL1, PCL1 \\
\hline $\operatorname{miR} 164$ & NAC, FK171, NILP3, FDL3 \\
\hline $\operatorname{miR} 166 \mathrm{i}$ & ATHB8, ATB15, REV, MOM1, EK1 \\
\hline $\operatorname{miR} 172$ & GATLA, SKP1, APY2, RHF2A, SYLM, PP413 \\
\hline miR390e & TIR1, TMKL1, Y4265 \\
\hline $\operatorname{miR} 319$ & NSP2, DET2, TCP4,SR542 \\
\hline $\operatorname{miR} 393$ & AFB2, NTPCR, ICE1, TIR1 \\
\hline $\operatorname{miR} 396$ & LU7L3, NACK2, TBCD, PPR15, Y3918, GRF4 \\
\hline $\operatorname{miR} 399$ & $\mathrm{UBC} 24$ \\
\hline $\operatorname{miR} 4376$ & ACA8, C78A3, DJC14 \\
\hline $\operatorname{miR} 4995$ & GIP, METE \\
\hline $\operatorname{miR6138}$ & CUL1, SPCS1, RDM4, TMVRN, NCS, Y2913, JAC1, PDAT1, LAZH2, MIRA \\
\hline $\operatorname{miR} 6140$ & APA1, Y5673, LTR3B, TSS, RMP, HSP70, CID9, PP344 \\
\hline $\operatorname{miR6143}$ & XPO7, CSK2B, MLO10 \\
\hline $\operatorname{miR6173}$ & GAF1 \\
\hline \multicolumn{2}{|l|}{ Novel miRNAs } \\
\hline comp17305_c0_seq 1 & GCP2, VIL2, FK111, AGO7 \\
\hline comp44202_c0_seq 1 & RP8L2 \\
\hline comp46638_c0_seq 1 & SPXM3, P2C16, B561A, ADCK4, AIL5, CSE, ZN363, SKS2 \\
\hline comp46638_c0_seq 1_star & M810, ATM, GBF4 \\
\hline comp48783_c0_seq 3 & APS3, RH24 \\
\hline comp62834_c0_seq 6_star & 5NTC, XYLL3, PTR18, KDEL1, GTE4, GDL76, S38A6, TRPC, CBSX6, PLST1 \\
\hline comp72313_c0_seq 1 & MNS5, BHO79, UGDH5 \\
\hline
\end{tabular}

was similar to the KEGG annotation of known miRNA target genes.

\section{miRNA targets validation by degradome sequencing}

To further understand the role of miRNA in $P$. ginseng, degradome sequencing of $P$. ginseng was used to identify miRNA targets. The reference gene sequence in which the degraded gene fragment is located is aligned with the NR, KEGG, and GO databases using Cleaveland 3 software to perform functional annotation of the target gene. Two target genes for miR159 were identified, namely GAM1_ORYSI and TCP24_ARATH. GAMYB transcription factor is an important transcription factor in the gibberellin signal transduction pathway, and TCP transcription factor is involved in various signal transduction pathways, connected growth and mediated stress response. miR393 targets AFB2_ARATH and TIR1_ARATH to altering the auxin distribution in plants by negative regulation of TIR1 (transport inhibitor response 1) and AFB2 (protein auxin signaling F-BOX). This was basically consistent with the target gene type in high throughput sequencing of small RNA. MiR396 participates in the growth process of ginseng by targeting the expression of GRF (growth-regulating factor) and protein auxin signaling (F-BOX 3). The target genes of the three novel miRNAs were detected to be associated with SPX factor and TIR1 disease resistance factor (Table 2).

\section{Discussion}

In this study, three high-throughput approaches, namely, transcriptomics, small RNA and degradome sequencing were used for better understanding of differences between Shizhu ginseng and Yuan ginseng. After the stringent quality filters and de novo assemble, a total of 63,875 unigenes were obtained. We have identified 3206 DEGs (1190 up-regulated and 2016 down-regulated). These 
A

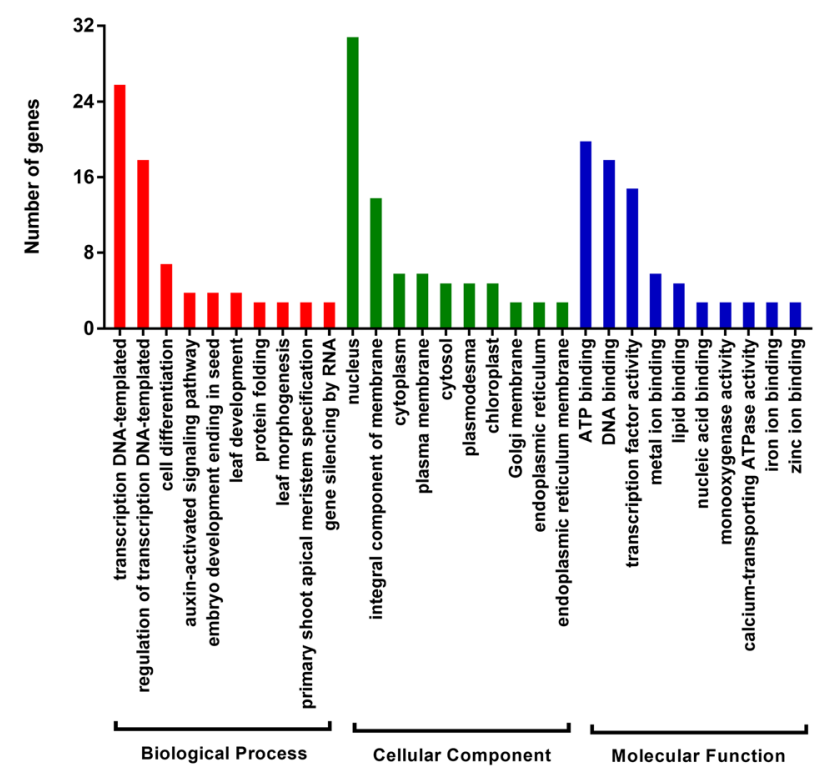

$\mathrm{C}$

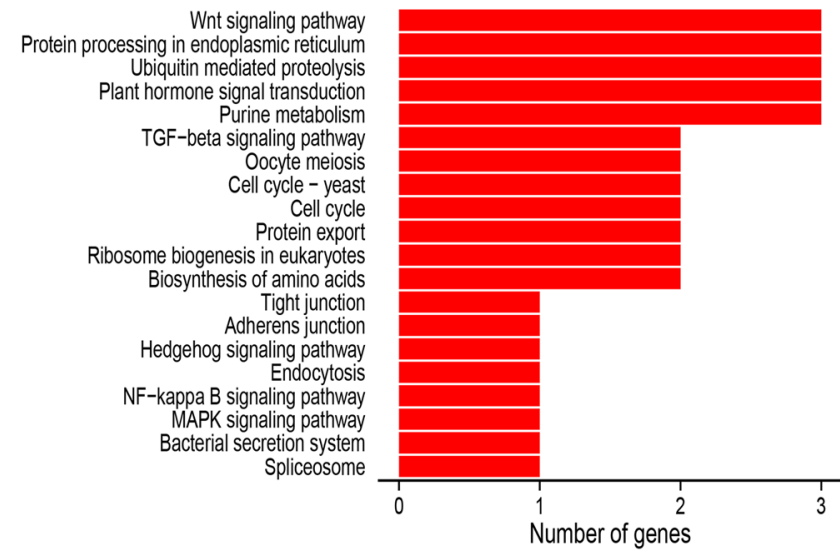

Fig. 7 GO and KEGG enrichment of target genes of DEMs. a GO enrichment analysis of target genes of known DEMs. b GO enrichment analysis of target genes of novel DEMs. c KEGG enrichment

DEGs mainly enriched in phenylpropanoid biosynthesis, biosynthesis of unsaturated fatty acids, apoplast, etc.

Plants are rich in miRNA, which can regulate plant growth and development, nutrient metabolism and stress response. For example, the target gene ICE1 (transcription factor of basic helix-loop-helix (bHLH) DNA-binding superfamily protein) of miR393 plays a role in cold tolerance by controlling cold-responsive genes (Ohta et al. 2018).

The regulation of some miRNA target genes is involved in the production process of plant secondary metabolites, which is conducive to the accumulation of pharmacodynamic substances. The target gene of miR159 is MYB
B

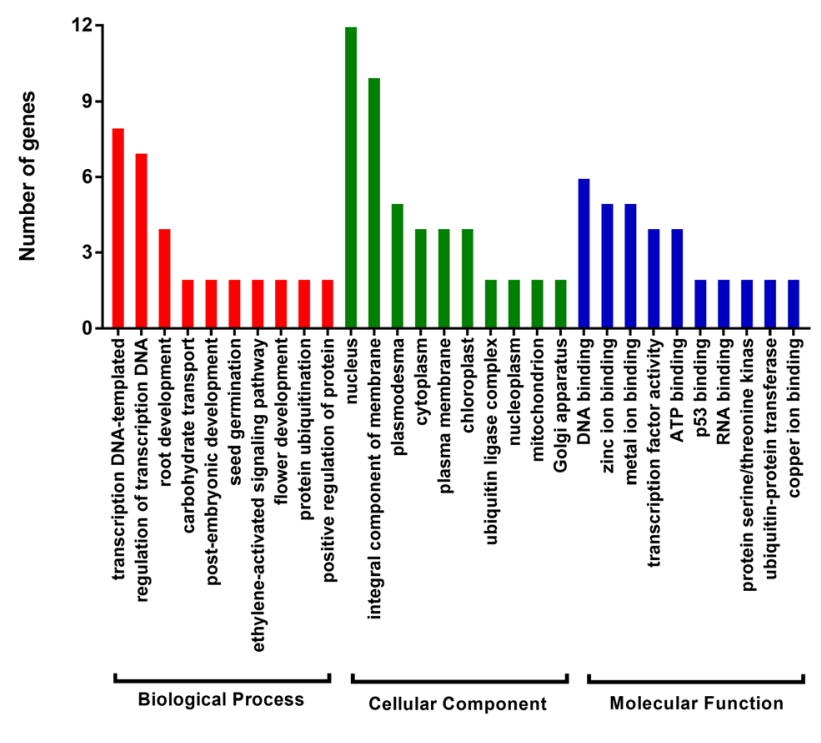

D

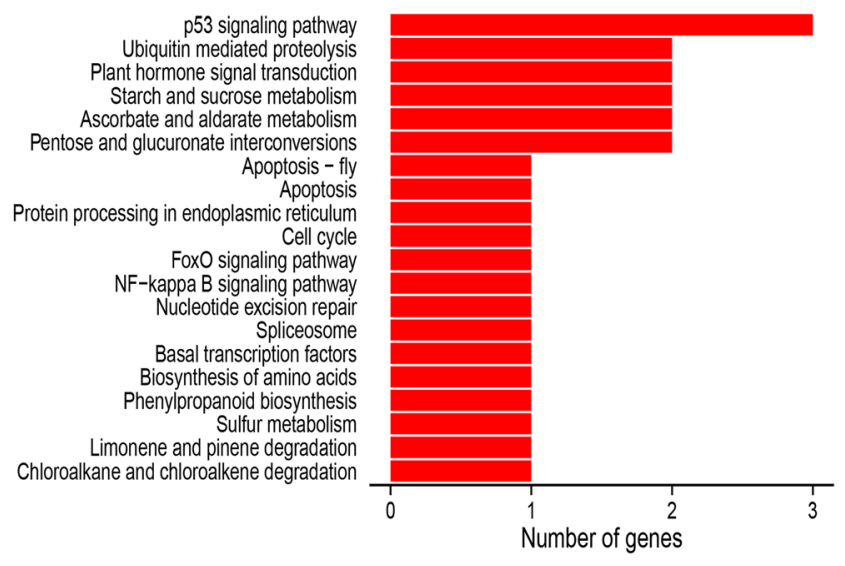

analysis of target genes of known DEMs. d KEGG enrichment analysis of target genes of novel DEMs

(Transcription factor MYB51), and the MYB transcription factor is involved in the biosynthesis of fat and glucosinolate (Frerigmann and Gigolashvili 2014). Glucosinolate is abiological compound with anti-cancer and anti-oxidant activity, and its hydrolysateisothiocyanate can prevent and treat various cancers, scavenge free radicals, protect cardiovascular and cerebrovascular, and delay aging (KonicRistic et al. 2016). The target gene of miR172 encodes the glycosyltransferase (GATLA),which is a member of a multi-gene family of plants that transfer single or multiple activated sugars to a range of plant molecules, resulting in glycosylation of plant compounds. The UDP glucosyltransferase gene $P$. ginseng is a key enzyme for the 
Table 2 The annotation of miRNAs in degradation sequencing

\begin{tabular}{lll}
\hline miRNA & Target & Target gene annotation \\
\hline miR159/319 & GAM1_ORYSI & Transcription factor GAMYB \\
miR160 & TCP24_ARATH & TCP transcription factor \\
miR164 & ARFR_ORYSJ & Auxin response factor \\
miR165/166 & NC100_ARATH & NAC domain-containing protein \\
miR172 & ATB15_ARATH & DNA binding protein \\
& AP2_ARATH & Ethylene-responsivetranscription factor \\
miR393 & RH46_ARATH & DEAD-box ATP-dependent RNA helicase \\
& AFB2_ARATH & Protein auxin signaling F-BOX \\
miR396 & TIR1_ARATH & Transport inhibitor response 1 \\
& GRF6_ORYSJ & Uncharacterized protein \\
miR399 & GRF3_ORYSJ & Growth-regulating factor 3 \\
miR4376 & UBC24_ARATH & Ubiquitin-conjugating enzyme E2 \\
comp46638 & ACA8_ARATH & Autoinhibited calcium ATPase \\
comp62834 & SPXM3_ARATH & SPX domain-containing membrane protein \\
comp65451 & SVP_ARATH & MADS-box protein SVP \\
\hline
\end{tabular}

synthesis of ginsenoside ( $\mathrm{Lu}$ et al. 2017), which is most likely to participate in triterpenoid synthesis (Fan et al. 2018; Khorolragchaa et al. 2014).

miRNA target genes are evolutionarily conserved among many known species. It was reported that plant miRNAs could transfer and regulate gene expression in a cross-kingdom manner, namely, affecting the organism from which they do not originate. Zhang Chenyu et al. fed fresh rice which contained abundant MIR168a to mice and found that MIR168a could bind to the exon 4 of low density lipoprotein receptor adapter protein 1 (LDLRAP1) mRNA and reduces its protein expression in vitro and in vivo, and consequently regulate the lipid metabolism of the mouse (Zhang et al. 2012). Zhang Chenyu et al. found that miR2911 contained in honeysuckle signifcantly inhibited H1N1-encoded PB2 and NS1 protein expression, and consequently played a direct role in the influenza virus in mice (Zhou et al. 2015). It has been found that the miRNA of dodder (Cuscuta spp.) can as a causative agent to target host mRNA across species to regulate its gene expression and play a role in the process of parasitization (Shahid et al. 2018). Plant miRNAs which were are more enriched in beebread than inroyal jelly can induce larval developed into worker bees by delaying development and decreasing body and ovary size (Zhu et al. 2017). It provides a basis for the cross-kingdom regulation of plant miRNAs.

Wang Yingfang et al. had detected abundant miRNAs in fresh ginseng and ginseng decoction (Wang et al. 2018; Wenjuan et al. 2017). It is speculated that ginseng miRNA may be one of the material basis for its clinical efficacy. The difference in clinical efficacy of ginseng with different growth years may be caused by the regulation of its intrinsic miRNA. Based on this, the research group carried out this study. Shizhu ginseng and Yuan ginseng are different varieties of Chinese ginseng, and their growth years differ by more than 10 years. The DEMs of Shizhu ginseng and Yuan ginseng were identified by high-throughput sequencing, and some related target genes were annotated.

In the experiment, there were 17 known miRNAs and 7 novel miRNAs with different in Shizhu ginseng and Yuan ginseng. GO and KEGG functional enrichment analysis was performed on targets of known and novel miRNA families. For GO functional enrichment, in the main category of "biological processes", the most significant GO terms were "regulation of transcription", "cell differentiation", and "auxin-activated signaling pathway". With respect to the main category of "cell component" class, the "nucleus", "integral component of membrane" and "cytoplasm" was the domain term. Under the third main category of "molecular function", the dominant terms were "binding", "catalytic activity", and "transcription factor activity". In functional KEGG analysis, target genes are enriched in plant hormone signal transduction, p53 signaling pathway, metabolism, and Ubiquitin mediated proteolysis.

Among them, the target genes of miR396 are LU7L3 (luc7-like protein 3 isoform X2) and TBCD (tubulin-folding cofactor D). LUC7L is defined at the centromeric border of the human alpha-globin domain and inhibits replication of hepatitis $\mathrm{B}$ virus by inhibiting enhancer II or basal core promoter activity (Li et al. 2016). TBCD encodes tubulin folding cofactor $\mathrm{D}$, a dynamic cytoskeletal component that coordinates and supports a variety of neuronal processes, including cell division, migration, intracellular trafficking, and signal transduction. Mutations 
or altered microtubule dynamics can cause neurodevelopmental disorders and neurodegenerative diseases (Flex et al. 2016; Miyake et al. 2016). Studies have shown that TBCD interacts physically with the intracellular domain of Down's syndrome cell adhesion molecule (Dscam), which is important for neural development (Okumura et al. 2015).

The novel miRNA comp46638 targets B561A (cytochrome b561, DM13 and DOMON domain-containing protein). B561A is found in various organs and cell types of plants and animals. Physiological functions of CYB561s include stress defense, cell wall modification, iron metabolism, tumor suppression, and various neural processes and memory retention (Asard et al. 2013). TBCD and B561A are involved in the process of neurodevelopment, which may be the target of ginseng miRNAs to play a stimulating role.

The novel miRNA comp65451 target gene is involved in the regulation of TIR NBS-LRR (Toll-interleukin-like receptor, nucleotide-binding site and C-terminal leucinerich repeat), and may be a novel miRNA specific for ginseng species.

The target of miR319 is SR542, which is highly conserved and exists in bacteriaand mammals. Autosomal dominant mutations in SRP54 are key factors in co-translational protein targeting pathways, leading to neutropenia like Shwachman-Diamond syndrome (Carapito et al. 2017).

The target of miR393, NTPCR, encodes a cancer-associated nucleoside phosphatase, a cancer-associated PRUNE2 protein that is nucleotide-associated and highly expressed in mature neural tissue, and its neural tissuespecific and post-developmental expression may help maintain maturity the nervous system (Iwama et al. 2011).

Some target genes indirectly exert anti-tumor effects, such as NTPCR, MYB, FDL3, etc., by proteins encoding cancer-associated proteins and secondary metabolites with anticancer effects. Chin AR et al. found that oral miR159 mimics significantly inhibited the growth of mouse xenograft breast tumors (Chin et al. 2016).

The above analysis shows that this study lays a foundation for further exploring the differences between Shizhu ginseng and Yuan ginseng and revealing the possible regulation of ginseng miRNA and its target genes.

Acknowledgements This work was supported by grants from the National Natural Science Foundation for Young Scholars of China (81403195) and the Natural Science Foundation of Guangdong Province (S2013010015418).

Author contributions YW, ZY, YZ and BH contributed to the study conception and design. Material preparation, data collection and analysis were performed by MP, YC, WW, ZH, WD, ZL, MG, and YY. The first draft of the manuscript was written by MP and YW and all authors commented on previous versions of the manuscript. All authors read and approved the final manuscript.

\section{Compliance with ethical standards}

Conflict of interest The authors declare that they have no confict of interest.

Open Access This article is licensed under a Creative Commons Attribution 4.0 International License, which permits use, sharing, adaptation, distribution and reproduction in any medium or format, as long as you give appropriate credit to the original author(s) and the source, provide a link to the Creative Commons licence, and indicate if changes were made. The images or other third party material in this article are included in the article's Creative Commons licence, unless indicated otherwise in a credit line to the material. If material is not included in the article's Creative Commons licence and your intended use is not permitted by statutory regulation or exceeds the permitted use, you will need to obtain permission directly from the copyright holder. To view a copy of this licence, visit http://creativecommons. org/licenses/by/4.0/.

\section{References}

Asard H, Barbaro R, Trost P, Berczi A (2013) Cytochromes b561: ascorbate-mediated trans-membrane electron transport. Antioxid Redox Sign 19:1026-1035

Bonnet E, He Y, Billiau K, Van de Peer Y (2010) TAPIR, a web server for the prediction of plant microRNA targets, including target mimics. Bioinformatics 26:1566-1568

Carapito R, Konantz M, Paillard C, Miao ZC, Pichot A, Leduc MS, Yang YP, Bergstrom KL, Mahoney DH, Shardy DL, Alsaleh G, Naegely L, Kolmer A, Paul N, Hanauer A, Rolli V, Muller JS, Alghisi E, Sauteur L, Macquin C, Morlon A, Sancho CS, AmatiBonneau P, Procaccio V, Mosca-Boidron AL, Marle N, Osmani N, Lefebvre O, Goetz JG, Unal S, Akarsu NA, Radosavljevic M, Chenard MP, Rialland F, Grain A, Bene MC, Eveillard M, Vincent M, Guy J, Faivre L, Thauvin-Robinet C, Thevenon J, Myers K, Fleming MD, Shimamura A, Bottollier-Lemallaz E, Westhof E, Lengerke C, Isidor B, Bahram S (2017) Mutations in signal recognition particle SRP54 cause syndromic neutropenia with Shwachman-Diamond-like features. J Clin Invest 127:4090-4103

Chin AR, Fong MY, Somlo G, Wu J, Swiderski P, Wu X, Wang SE (2016) Cross-kingdom inhibition of breast cancer growth by plant miR159. Cell Res 26:217-228

Chunlu S, Wenzhong H, Cheng C, Jing L, Lu S (2016) Research progress of ginsenosides and their functional properties in Shizhu Ginseng. Chin Tradit Patent Med 38:890-893

Cock PJA, Fields CJ, Goto N, Heuer ML, Rice PM (2010) The Sanger FASTQ file format for sequences with quality scores, and the Solexa/Illumina FASTQ variants. Nucl Acids Res 38:1767-1771

Dai X, Zhuang Z, Zhao PX (2018) psRNATarget: a plant small RNA target analysis server (2017 release). Nucl Acids Res 46:W49W54

Dong HF, Lei JP, Ding L, Wen YQ, Ju HX, Zhang XJ (2013) MicroRNA: function, detection, and bioanalysis. Chem Rev 113:6207-6233

Fan B, Dong WX, Chen TY, Chu JL, He BF (2018) Switching glycosyltransferase UGT(BL)1 regioselectivity toward polydatin synthesis using a semi-rational design. Org Biomol Chem 16:2464-2469

Flex E, Niceta M, Cecchetti S, Thiffault I, Au MG, Capuano A, Piermarini E, Ivanova AA, Francis JW, Chillemi G, Chandramouli B, Carpentieri G, Haaxma CA, Ciolfi A, Pizzi S, 
Douglas GV, Levine K, Sferra A, Dentici ML, Pfundt RR, Le Pichon JB, Farrow E, Baas F, Piemonte F, Dallapiccola B, Graham JM, Saunders CJ, Bertini E, Kahn RA, Koolen DA, Tartaglia M (2016) Biallelic mutations in TBCD, encoding the tubulin folding cofactor $\mathrm{D}$, perturb microtubule dynamics and cause early-onset encephalopathy. Am J Hum Genet 99:962-973

Frerigmann H, Gigolashvili T (2014) MYB34, MYB51, and MYB122 distinctly regulate indolic glucosinolate biosynthesis in Arabidopsis thaliana. Mol Plant 7:814-828

Garg V, Khan AW, Kudapa H, Kale SM, Chitikineni A, Sun QW, Sharma M, Li CY, Zhang BH, Xin L, Kishor PBK, Varshney RK (2019) Integrated transcriptome, small RNA and degradome sequencing approaches provide insights into Ascochyta blight resistance in chickpea. Plant Biotechnol J 17:914-931

Iwama E, Tsuchimoto D, Iyama $T$, Sakumi $K$, Nakagawara A, Takayama K, Nakanishi Y, Nakabeppu Y (2011) Cancer-related PRUNE2 protein is associated with nucleotides and is highly expressed in mature nerve tissues. J Mol Neurosci 44:103-114

Keqiang L, Zhendong X, Hanlian W, Yuning S, Xin G (2013) Comparison study on chemical composition of Chinese Shizhu Panax, ginseng under forest and traditional Ginseng. Liaoning J Tradit Chin Med 40:423-425

Khorolragchaa A, Kim YJ, Rahimi S, Sukweenadhi J, Jang MG, Yang DC (2014) Grouping and characterization of putative glycosyltransferase genes from Panax ginseng Meyer. Gene 536:186-192

Konic-Ristic A, Stanojkovic T, Srdic-Rajic T, Dilber S, Dordevic B, Stankovic I, Juranic Z (2016) In vitro assessment of antiproliferative action selectivity of dietary isothiocyanates for tumor versus normal human cells. Vojnosanit Pregl 73:636-642

Kvam VM, Lu P, Si YQ (2012) A comparison of statistical methods for detecting differentially expressed genes from RNA-seq data. Am J Bot 99:248-256

Li Y, Ito M, Sun S, Chida T, Nakashima K, Suzuki T (2016) LUC7L3/CROP inhibits replication of hepatitis B virus via suppressing enhancer II/basal core promoter activity. Sci Rep 6(1):1-11

Lu C, Zhao SJ, Wang XS (2017) Functional regulation of a UDPglucosyltransferase gene (Pq3-O-UGT1) by RNA interference and overexpression in Panax quinquefolius. Plant Cell Tiss Org $129: 445-456$

Mackowiak SD (2011) Identification of novel and known miRNAs in deep-sequencing data with miRDeep2. Curr Protoc Bioinform 36(1):12

Mathiyalagan R, Subramaniyam S, Natarajan S, Kim YJ, Sun MS, Kim SY, Kim YJ, Yang DC (2013) Insilico profiling of microRNAs in Korean ginseng (Panax ginseng Meyer). J Ginseng Res 37:227-247

Miyake N, Fukai R, Ohba C, Chihara T, Miura M, Shimizu H, Kakita A, Imagawa E, Shiina M, Ogata K, Okuno-Yuguchi J, Fueki N, Ogiso Y, Suzumura H, Watabe Y, Imataka G, Leong HY, FattalValevski A, Kramer U, Miyatake S, Kato M, Okamoto N, Sato Y, Mitsuhashi S, Nishino I, Kaneko N, Nishiyama A, Tamura T, Mizuguchi T, Nakashima M, Tanaka F, Saitsu H, Matsumoto N
(2016) Biallelic TBCD mutations cause early-onset neurodegenerative encephalopathy. Am J Hum Genet 99:950-961

Ohta M, Sato A, Renhu N, Yamamoto T, Oka N, Zhu JK, Tada Y, Suzaki T, Miura K (2018) MYC-type transcription factors, MYC67 and MYC70, interact with ICE1 and negatively regulate cold tolerance in Arabidopsis. Sci Rep 8:11622

Okumura M, Sakuma C, Miura M, Chihara T (2015) Linking cell surface receptors to microtubules: tubulin folding cofactor D mediates Dscam functions during neuronal morphogenesis. J Neurosci 35:1979-1990

Rajabian A, Rameshrad M, Hosseinzadeh H (2019) Therapeutic potential of Panax ginseng and its constituents, ginsenosides and gintonin, in neurological and neurodegenerative disorders: a patent review. Expert Opin Ther Pat 29:55-72

Shahid S, Kim G, Johnson NR, Wafula E, Wang F, Coruh C, BernalGaleano V, Phifer T, dePamphilis CW, Westwood JH, Axtell MJ (2018) MicroRNAs from the parasitic plant Cuscuta campestris target host messenger RNAs. Nature 553:82

Wang M, Wu B, Chen C, Lu S (2015) Identification of mRNA-like non-coding RNAs and validation of a mighty one named MAR in Panax ginseng. J Integr Plant Biol 57:256-270

Wang Y-F, Wang W-J, Chen Y-L, He Z-H, Cao J-J, Yang Z-M, Gong M-J, Yin Y-Q (2018) Extraction and verification of miRNA from ginseng decoction. Chin Herb Med 10:318-322

Wenjuan W, Zhihua H, Yanlin C, Jingjing C, Zemin Y, Mengjuan G, Yongqin Y, Bin H, Yingfang W (2017) Comparison of two miRNA methods for extraction of Ginseng Decoction. J Guangdong Pharm Univ 33:595-599

Ying-fang W, Yan-lin C, Wen-juan W (2019) Research progress of miRNAs in traditional Chinese medicines. Chin J New Drugs 28:432-436

Zhang ZH, Yu JY, Li DF, Zhang ZY, Liu FX, Zhou X, Wang T, Ling Y, Su Z (2010) PMRD: plant microRNA database. Nucl Acids Res 38:D806-D813

Zhang L, Hou DX, Chen X, Li DH, Zhu LY, Zhang YJ, Li J, Bian Z, Liang XY, Cai X, Yin Y, Wang C, Zhang TF, Zhu DH, Zhang DM, Xu J, Chen Q, Ba Y, Liu J, Wang Q, Chen JQ, Wang J, Wang M, Zhang QP, Zhang JF, Zen K, Zhang CY (2012) Exogenous plant MIR168a specifically targets mammalian LDLRAP1: evidence of cross-kingdom regulation by microRNA (vol 22, pg 273, 2012). Cell Res 22:273-274

Zhou Z, Li XH, Liu JX, Dong L, Chen Q, Liu JL, Kong HH, Zhang QY, Qi X, Hou DX, Zhang L, Zhang GQ, Liu YC, Zhang YJ, Li J, Wang J, Chen X, Wang H, Zhang JF, Chen HL, Zen K, Zhang CY (2015) Honeysuckle-encoded atypical microRNA2911 directly targets influenza A viruses. Cell Res 25:39-49

Zhu KA, Liu MH, Fu Z, Zhou Z, Kong Y, Liang HW, Lin ZG, Luo J, Zheng HQ, Wan P, Zhang JF, Zen K, Chen J, Hu FL, Zhang CY, Ren J, Chen X (2017) Plant microRNAs in larval food regulate honeybee caste development. PLoS Genet 13:e1006946

Publisher's Note Springer Nature remains neutral with regard to jurisdictional claims in published maps and institutional affiliations. 